\title{
EVOLUCIÓN DEL CONCEPTO DE USABILIDAD COMO INDICADOR DE CALIDAD DEL SOFTWARE
}

\author{
José-Luis González-Sánchez, Francisco Montero-Simarro y Francisco-Luis \\ Gutiérrez-Vela
}
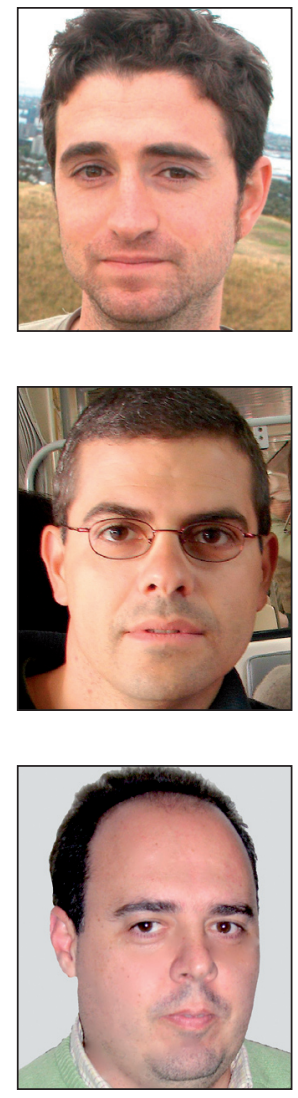

José-Luis González-Sánchez es doctor y profesor del Depto. de Informática e Ing. Industrial de la Esc. Politécnica Sup. de la Univ. de Lleida (UDL). Imparte docencia en los estudios de Grado en Informática dentro de la especialización de ingeniería del software y colabora como profesor en diferentes masters oficiales en la UDL y en la Univ. de La Laguna. Miembro de la Asoc. Española de Interacción Persona Ordenador y de la Academia de Artes y Ciencias Interactivas. Investiga en la caracterización y evaluación de la experiencia del usuario en sistemas interactivos.

Univ. de Lleida, Depto. de Informática e Ing. Industrial Jaume II, 69. 25071 Lleida, España joseluisgs@diei.udl.cat

Francisco Montero-Simarro es doctor y profesor en el Depto. de Sistemas Informáticos de la Univ. de Castilla-La Mancha. Imparte docencia en los estudios de Grado en Informática dentro de la especialización de ingeniería del software y colabora como profesor en masters oficiales en la Univ. de Castilla-La Mancha y en la Univ. de Lleida. Miembro de la Asoc. Española de Interacción Persona-Ordenador. Investiga sobre el logro y la caracterización de la calidad de la interacción entre hombre y máquina.

Univ. de Castilla-La Mancha, Depto. de Sistemas Informáticos Campus Universitario, s/n. 02071 Albacete, España

fmontero@dsi.uclm.es

Francisco-Luis Gutiérrez-Vela es doctor y profesor en el Depto. de Lenguajes y Sistemas Informáticos de la Univ. de Granada (UGR). Imparte docencia en los estudios de Grado en Informática dentro de la especialización de Ingeniería del Software y también colabora como profesor en masters oficiales en la UGR y en la Univ. de La Laguna. Miembro de la Asoc. Española de Interacción Persona-Ordenador. Investiga sobre sistemas interactivos.

Univ. de Granada, Depto. de Lenguajes y Sistemas Informáticos Periodista Daniel Saucedo, s/n. 18071 Granada, España fgutierr@ugr.es

\section{Resumen}

Los constantes avances tecnológicos en el sector de los sistemas interactivos y de información hacen que cada vez se consideren más factores y criterios para valorar su calidad durante su diseño, desarrollo y evaluación. Conceptos como la calidad en uso o la experiencia de usuario han entrado en escena y en muchas ocasiones aparecen sustituyendo o acompañando a la usabilidad en presentaciones, informes y artículos. Se identifica y analiza la evolución de estos conceptos y se caracteriza y sitúa cada factor de calidad en una dimensión y con un alcance determinado. Se identifican igualmente las relaciones y solapamientos entre esos mismos factores.

\section{Palabras clave}

User friendliness, Usabilidad, Calidad en uso, Experiencia de usuario, Sistemas interactivos, Sistemas de información.

\section{Title: The evolution of the usability concept as an indicator of software quality}

\begin{abstract}
Technological advances in the field of information and interactive systems mean that increasing numbers of factors and criteria are considered to assess quality during the design, development and evaluation process. In this context, concepts such as quality of use and user experience have appeared and in many cases are replacing or supplementing existing usability properties to identify the quality of the interaction process. In this paper we describe and analyze the evolution of the different concepts to identify the quality of the interaction on information systems and we help to characterize and locate each quality factor in one dimension and a specific range of the process of use, and consider the relationships and overlap between these concepts.
\end{abstract}




\section{Keywords}

User friendliness, Usability, Quality in use, User experience, Interactive systems, Information systems.

González-Sánchez, José-Luis; Montero-Simarro, Francisco; Gutiérrez-Vela, Francisco-Luis. "Evolución del concepto de usabilidad como indicador de calidad del software". El profesional de la información, 2012, septiembre-octubre, v. 21, n. 5 , pp. 529-536

http://dx.doi.org/10.3145/epi.2012.sep.13

\section{Introducción}

La calidad de los sistemas informáticos depende de múltiples factores y criterios, que se vienen proponiendo en lo que se conoce como modelos de calidad del software (ISO/ IEC 9126:1991; ISO/IEC 9126-1:2001). Estos modelos especifican características, factores y métricas que dichos sistemas deberían tener, y están basados en la idea de que el logro de la calidad depende a su vez de la calidad con la que esos sistemas fueron desarrollados.

La calidad de la interacción influye de manera decisiva en que los usuarios se decanten por un sistema u otro

Hay múltiples modelos de calidad disponibles en la bibliografía. Por ejemplo las propuestas de McCall, Richards y Walters (1977); Boehm et al. (1978), Dromey (1996); otras sobre la calidad de la interacción con el usuario a través de la consideración de factores humanos (Norman, 2002), criterios ergonómicos (Bastien; Scapin, 1995) o conceptos como el de la usabilidad (Nielsen, 1993; ISO 9241-11:1998; ISO/IEC 9126-1:2001). En muchos casos este concepto se ha usado para hacer referencia a la facilidad de uso de un sistema, pero ni esa era la única característica que aportaba ni su caracterización puede decirse en la actualidad que esté totalmente cerrada. Más bien al contrario, progresivamente se observa que el concepto de usabilidad se está utilizando con una mayor carga de atributos. Además aparecen otros conceptos en situaciones de uso parecidas, haciendo más difuso su contexto de aplicación.

La presencia de estándares internacionales en unas ocasiones, y de facto en otras, ha contribuido a poner cierto orden en este escenario de definiciones solapadas. Aun así distintas disciplinas, no siempre con los mismos intereses ni objetivos, tienden a utilizar los mismos conceptos con múltiples acepciones, consideraciones y dimensiones. Se hace necesaria una reflexión que trate de contribuir al uso apropiado y consciente de lo que cada característica de calidad aporta.

El objetivo fundamental de este trabajo es contribuir a analizar desde el punto de vista del usuario final, qué características de calidad se han considerado a la hora de desarrollar sistemas software. En función de este objetivo la organización del presente artículo es la siguiente: en primer lugar, se identificará qué es y cuál es el alcance de un modelo de calidad. Después pasaremos a identificar las características de la calidad más dependientes de la percepción del usuario final. En ese ámbito la usabilidad es el concepto que viene ocupando el lugar más destacado, pero se observa que otros términos recién aparecidos y cada día más utilizados aportan criterios adicionales que también influyen en la calidad software que percibe el usuario. A continuación se definen y caracterizan esos conceptos alternativos; concretamente, la calidad en uso y la experiencia de usuario. Por último se analiza el escenario actual y se pronostica una evolución en el uso de los términos considerados.

\section{Modelos de calidad de software}

La calidad de un sistema interactivo y de información tiene múltiples visiones y su logro supone un esfuerzo y un reto en la aplicación de técnicas, metodologías y recursos. Desde este punto de vista, la calidad de un sistema es la conformidad de requisitos funcionales y no funcionales documentados explícitamente, así como las características implícitas que se espera que todo software desarrollado profesionalmente debe cumplir (Pressman, 1997).

La definición anterior pone de manifiesto la necesidad de caracterizar en base a requisitos y características los atributos deseables explícita o implícitamente de un sistema. Es ahí donde surge la necesidad de descomponer la calidad en un conjunto de características. Dichas características serían de orden inferior y progresivamente deberían ser más fáciles de estimar o evaluar.

\section{La percepción que un usuario tiene de un sistema interactivo y de información está influenciada por cada vez más aspectos del contexto y del estado anímico}

En la literatura existen múltiples propuestas de descomposición de la calidad. Existen también distintas recomendaciones para la elaboración de modelos de calidad. Quizá una de las más conocidas y extendidas es la de Basili et al. (2007) denominada GQM, pero lo que tienen en común todas ellas es la presencia de una calidad ligada al uso que el usuario final de un sistema hace de él.

Otra característica común en los modelos de calidad es su disposición jerárquica (figura 1). Se habla de factores, criterios y métricas, o de características, subcaracterísticas y métricas para referirse a los distintos elementos que aparecen en la descomposición.

Los primeros modelos de calidad de la Organización Internacional de Estandarización (ISO) han identificado seis características: funcionalidad, eficiencia, usabilidad, fiabilidad, facilidad de mantenimiento y facilidad de portabilidad. Di- 


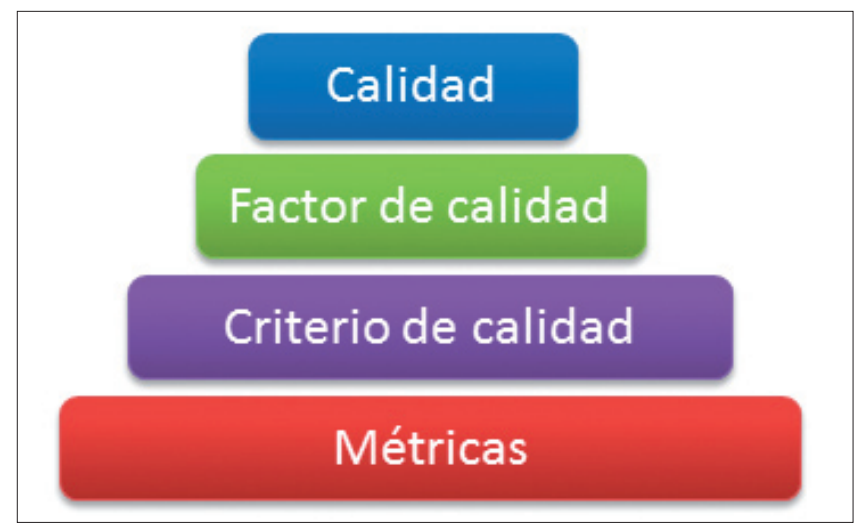

Figura 1. Estructura de un modelo de calidad

chos factores se identifican por ejemplo en los estándares ISO/IEC 9126:1991, y en su revisión ISO/IEC 9126-1:2001.

Recientemente se ha propuesto un nuevo modelo de calidad (ISO/IEC 25010:2011) incluido dentro de la norma ISO/IEC 25000:2005: SQuaRE (Software product quality requirements and evaluation). En él no sólo pasan de seis a ocho las características ligadas a un sistema (funcionalidad, eficiencia, compatibilidad, usabilidad, fiabilidad, seguridad, facilidad de mantenimiento y portabilidad), sino que además una nueva visión de calidad se identifica y define: la calidad de la interacción, es decir, la relacionada con los resultados de la interacción con un sistema expresados en términos de efectividad, eficiencia, satisfacción, protección frente a riesgos y cobertura.

El nivel y grado de detalle con el que se han ido presentando los modelos de calidad disponibles cada vez trata de ser más preciso y van acumulándose nuevos criterios y factores que contribuyen a esa precisión.

\section{Pasado y presente de la usabilidad}

La usabilidad es un concepto conocido y utilizado. Proviene de lo que se denominaba user friendly. Con este término se hacía hincapié en la facilidad de manejo que ofrecía un sistema a través de su interfaz. Definiciones de distintos modelos de usabilidad pueden encontrase en Norman (2002), Nielsen (1993), Mayhew (1999), Constantine y Lockwood (1999). Otras definiciones surgieron de diferentes estándares internacionales que aglutinaron consensos en su definición (ISO 9241-11:1998, ahora ISO 9241-211), y la ISO/IEC 9126-1:2001, donde se define la usabilidad atendiendo a dos puntos de vista: como proceso y como producto (figura 2).

\begin{tabular}{|l|l|}
\hline \multicolumn{1}{|c|}{ Criterio } & \multicolumn{1}{c|}{ Métrica } \\
\hline Efectividad & $\begin{array}{l}\text { Porcentaje de objetivos logrados } \\
\text { Porcentaje de usuarios que completaron } \\
\text { satisfactoriamente las tareas } \\
\text { Media de precisión al completar las tareas }\end{array}$ \\
\hline Eficiencia & $\begin{array}{l}\text { Tiempo para completar una tarea } \\
\text { Tareas completadas por unidad de tiempo } \\
\text { Coste económico de realización de la tarea }\end{array}$ \\
\hline Satisfacción & $\begin{array}{l}\text { Tasa de escala de satisfacción } \\
\text { Tasa de uso en el tiempo } \\
\text { Frecuencia de quejas }\end{array}$ \\
\hline
\end{tabular}

Tabla 1. Ejemplos de métricas de usabilidad (ISO 9241-11)

\section{Usabilidad como producto}

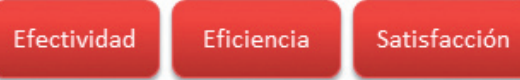

Usabilidad como proceso

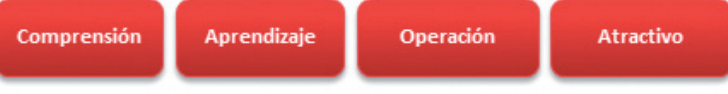

Figura 2. La usabilidad como producto y como proceso

La usabilidad vista como proceso presenta métricas para su evaluación que dependen de la efectividad, eficiencia y satisfacción. Mientras que vista como producto descansa en la facilidad para aprender, recordar, y operar con dicho producto, además de resultar atractivo. En los estándares mencionados se proponen métricas para una y otra visón.

Obviamente las conexiones entre una y otra definición son múltiples, pero no están demostradas ni validadas, y además cuentan con adeptos y detractores en según qué disciplinas. Por ejemplo la primera de las normas es ampliamente aceptada en la comunidad de Interacción Persona-Ordenador, mientras que la segunda cuenta con mayor número de seguidores entre los pertenecientes a la disciplina de Ingeniería del Software.

\subsection{Usabilidad como proceso}

Se define como la eficacia, eficiencia y satisfacción con la que un sistema permite alcanzar objetivos específicos a usuarios concretos en un contexto de uso también específico (ISO 9241-11:1998).

La definición anterior es idónea para evaluar sistemas software o de información, por ejemplo, en un ámbito de exigencia profesional, pero presenta limitaciones cuando la efectividad o la eficiencia no son las características más demandadas, por ejemplo cuando estamos tratando con productos destinados al entretenimiento en los que la dimensión satisfacción juega un papel más relevante.

\subsection{Usabilidad como producto}

Es la capacidad de un software de ser comprendido, aprendido, usado y que resulte atractivo para el usuario en condiciones específicas de uso (ISO/IEC 9126-1:2001). En la tabla 2 se muestran métricas internas (inherentes a los elementos del producto software), y externas (cuando el sistema es utilizado por usuarios).

En cualquier caso la usabilidad queda presente como medida genérica de calidad de los sistemas de información, como se ha podido demostrar en diversos estudios y proyectos que hacen uso de ella como elemento identificador de la calidad del software, muchos de estos estudios publicados en esta revista, como Ribera, Térmens y García-Martín, (2008), Baeza-Yates, Rivera-Loaiza y Velasco-Martín (2004) o Mar$\cos (2004)$. 


\begin{tabular}{|c|c|c|}
\hline Usabilidad & Internas (ISO/IEC TR 9126-3:2003) & Externas (ISO/IEC TR 9126-2:2003) \\
\hline $\begin{array}{l}\text { Fácil de } \\
\text { comprender }\end{array}$ & $\begin{array}{l}\text { Funciones documentadas } \\
\text { Funciones con demostrador } \\
\text { Funciones evidentes } \\
\text { Comprensión de funciones }\end{array}$ & $\begin{array}{l}\text { Funciones entendidas después de leer su descripción } \\
\text { Demostraciones/tutoriales } \\
\text { Tutoriales durante la operación } \\
\text { Efectividad de la demostración. }\end{array}$ \\
\hline Fácil de aprender & Completitud de la ayuda/asistencia & $\begin{array}{l}\text { ¿Cuánto tiempo cuesta a un usuario aprender a utilizar una función? } \\
\text { ¿Cuánto tiempo cuesta aprender a un usuario a realizar una tarea de manera eficiente? } \\
\text { Tareas que pueden ser completadas correctamente, una vez se ha consultado la ayuda }\end{array}$ \\
\hline Fácil de operar & $\begin{array}{l}\text { Entradas validadas } \\
\text { Funciones cancelables } \\
\text { Funciones que se pueden deshacer } \\
\text { Funciones personalizables } \\
\text { Accesibilidad física } \\
\text { Claridad de los mensajes }\end{array}$ & $\begin{array}{l}\text { Nivel de consistencia de las componentes de interfaz } \\
\text { Errores corregidos una vez el sistema ha informado de ellos } \\
\text { Recuperación ante errores } \\
\text { Acierto en la introducción de datos } \\
\text { Mensajes entendidos }\end{array}$ \\
\hline
\end{tabular}

Tabla 2. Métricas relacionadas con la usabilidad vista como producto (adaptado de ISO/IEC 9126-3 y 9126-2).

\subsection{De la usabilidad a la calidad de la interacción}

En el estándar ISO/IEC TR 9126-4:2004 aparece y se oficializa el concepto de calidad en uso (Bevan, 1999a; 1999b). Dicho concepto viene a definirse en los mismos términos que la usabilidad, pero añade criterios de calidad nuevos como son la seguridad (safety), además de otras características que se han ido añadiendo en revisiones posteriores.

Sin embargo la reciente norma internacional incluida en el estándar denominado SQuaRE, (ISO/IEC 25010:2011), viene a eliminar la única y tradicional visión de la calidad de un producto software que venía recogiéndose en los estándares, y otorga a la calidad en uso una entidad propia, considerándola el factor determinante a nivel de calidad de la interacción. La calidad pasa a mostrarse dual: del sistema, dependiente de sus características y elementos propios, y de la interacción o calidad en uso (figura 3).

\begin{tabular}{|c|c|c|c|c|}
\hline \multicolumn{4}{|c|}{ Calidad en uso } & \multirow[b]{2}{*}{$\begin{array}{c}\text { Adecuación al } \\
\text { contexto }\end{array}$} \\
\hline Efectividad & Eficiencia & Satisfacción & $\begin{array}{l}\text { Libre de } \\
\text { riesgos }\end{array}$ & \\
\hline & & $\begin{array}{l}\text { - Utilidad } \\
\text { - Confianza } \\
\text { - Placer } \\
\text { - Comodidad }\end{array}$ & $\begin{array}{l}\text { - Mitigación de } \\
\text { los riesgos } \\
\text { económicos } \\
\text { - Mitigación de } \\
\text { los riesgos de } \\
\text { salud } \\
\text { - Mitigación de } \\
\text { los riesgos } \\
\text { ambientales }\end{array}$ & $\begin{array}{l}\text { - Completitud } \\
\text { al contexto } \\
\text { - Flexibilidad }\end{array}$ \\
\hline
\end{tabular}

Figura 3. Caracterización de la calidad en uso

La calidad en uso engloba a la usabilidad a través de muchos de sus factores. Eso sí, considerando cuando el producto software está en uso (efectividad en uso, eficiencia y satisfacción), y aporta como novedad la consideración de riesgos (freedom from risk) y la cobertura o adecuación del contexto de uso (context coverage), factores que no eran tenidos en cuenta en los modelos de usabilidad anteriormente comentados. Este nuevo estándar cubre aspectos indicadores de calidad anteriormente no considerados en otros estándares, como la accesibilidad y la adaptación de los contenidos al usuario (flexibilidad, personalización de contenidos, etc.). Las métricas comúnmente ofrecidas para evaluar la calidad

\begin{tabular}{|l|l|}
\hline \multicolumn{1}{|c|}{ Criterio } & \multicolumn{1}{c|}{ Métrica } \\
\hline Efectividad & $\begin{array}{l}\text { Efectividad de las tareas } \\
\text { Completitud de las tareas } \\
\text { Frecuencia de errores }\end{array}$ \\
\hline Eficiencia/ & $\begin{array}{l}\text { Tiempo de realización de la tarea } \\
\text { Eficiencia de la tarea } \\
\text { Productividad } \\
\text { Proporción productiva } \\
\text { Eficiencia de usuario relativo }\end{array}$ \\
\hline Protección & $\begin{array}{l}\text { Protección y salud del usuario } \\
\text { Protección de la gente afectada por la utilización } \\
\text { del sistema } \\
\text { Daño económico } \\
\text { Daño software }\end{array}$ \\
\hline Satisfacción & $\begin{array}{l}\text { Escala de satisfacción } \\
\text { Cuestionario de satisfacción } \\
\text { Uso discrecional }\end{array}$ \\
\hline
\end{tabular}

Tabla 3. Métricas relacionadas con la calidad en uso (ISO/IEC 9126-4:2004) en uso son las aportadas en el estándar ISO/IEC TR 9126-4:2004 (tabla 3).

Sin embargo es en la propiedad de la satisfacción donde este estándar da un paso más, intentando formalizar cuestiones hedónicas que estaban presentes en el análisis de la calidad del sistema y acercando dicha evaluación de la calidad a un proceso formal de medición de la experiencia del usuario (Law; Van-Schaik, 2010).

\section{Calidad de la interacción y experiencia del usuario}

La percepción que un usuario tiene de un sistema interactivo y de información está influenciada cada vez por más aspectos, tanto internos como externos al mismo, su uso, contexto y del estado anímico. Estas propiedades son determinantes en la experiencia o sensaciones al usarlo (Garrett, 2010; Law et al., 2008).

La experiencia de usuario (UX) se define como "la percepción de una persona y las respuestas subjetivas de esta como resultado de la utilización y/o el uso de un producto, sistema o servicio" (ISO/IEC 9241-210:2010). Está ligada al conjunto de sentimientos y respuestas emocionales que se producen 
en el usuario al interactuar con un producto software (Law; Van-Schaik, 2010; Law et al., 2009; Krug, 2005).

En la UX toman protagonismo los aspectos subjetivos o hedónicos de la calidad de uso debido a la propia evolución del concepto de satisfacción. En los enfoques tradicionales la percepción de "satisfacción" era analizada según la eficacia y eficiencia, de modo que si los usuarios perciben como eficaz y eficiente el uso del producto, se da por hecho que están satisfechos (Hassenzahl, 2003). Pero hay aspectos de la UX, como placer, confort, o utilidad, que contribuyen de manera significativa a la satisfacción del usuario con el sistema. Factores que han llegado a formar parte del nuevo estándar de la calidad en uso ISO/IEC 25010. Debemos remarcar la importancia de las campañas de marketing, el tratamiento al usuario por parte de los servicios de atención al cliente, los aspectos sociales ligados al uso de los productos, los comentarios y recomendaciones que llegan al usuario desde otros usuarios o comunidades.

La usabilidad es "absorbida" dentro de la UX, que es una forma elaborada de satisfacción (una de las tres propiedades básicas de la usabilidad)

Hay que considerar dos aspectos: en primer lugar, la usabilidad es 'absorbida' dentro de la UX; en segundo lugar, UX es una forma elaborada de satisfacción (una de las tres propiedades básicas de la usabilidad). La usabilidad es utilizada para medir y evaluar los objetivos pragmáticos (orientados a cómo el usuario realiza la tarea), mientras la UX (como forma elaborada de la satisfacción) se centra en la evaluación de los objetivos hedónicos (cómo se relaciona, siente y aprecia el usuario la realización de una tarea determinada) (Cockton, 2008). En ambos casos estas vertientes se intentan cubrir dentro del nuevo estándar de la calidad en uso ISO/IEC 25010 (figura 4).

A nivel de métricas los aspectos pragmáticos quedan cubiertos en gran parte por la usabilidad y la calidad en uso. El nuevo reto está en identificar, evaluar y detectar los aspectos hedónicos del proceso interactivo, y cómo poder diseñar un sistema que haga al usuario "sentirse bien" y cree en él "afinidad", "fidelidad" o "complacencia", aparte de que el grado de belleza estética, diseño visual, colores o estilo de la marca sean del agrado del usuario. Para ello se está in-

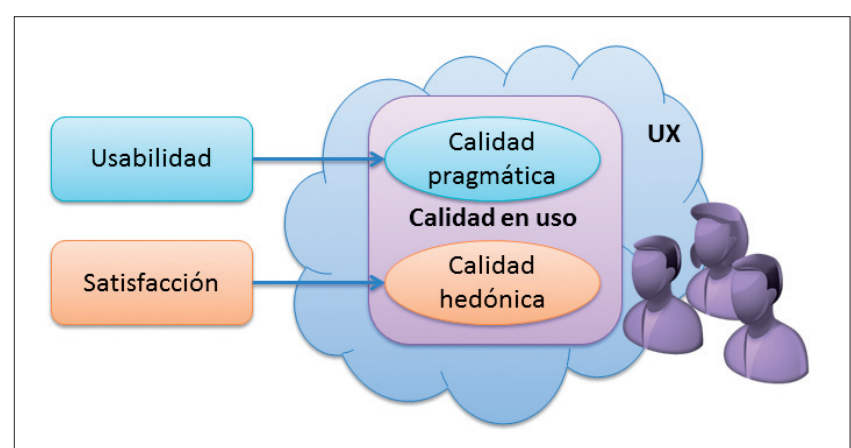

Figura 4. Caracterización de la experiencia del usuario a través de la calidad en uso dagando en técnicas de detección de emociones (respuesta emocional del usuario ante el proceso interactivo) (Norman, 2002; 2009) y análisis de la influencia multicultural en los usuarios de determinados sistemas (las personas se diferencian por un conjunto de aspectos culturales diferenciadores entre sociedades y por características internas propias) (Hofstede; Hofstede, 2005; Hassenzahl, 2003).

Las relaciones, atributos y evolución que identificamos entre los conceptos de calidad analizados en este artículo se muestran gráficamente en la figura 5 . En ella se atiende al grado de consideración del usuario (eje $\mathrm{X}$ ) y al grado de consideración del nivel de interacción (eje Y). En cada celda se han incluido algunas de las principales características identificadas y asociadas para cada concepto ligado a la calidad de la interacción, es decir, desde user-friendly a experiencia de usuario, pasando por usabilidad y calidad en uso. Identificamos igualmente una relación de inclusión entre cada factor en el sentido de abajo hacia arriba y de izquierda a derecha. La inclusión de los diferentes atributos en las distintas celdas no ha sido arbitraria sino que se ha llevado a cabo un estudio en el que han participado 20 profesionales relacionados con los distintos conceptos considerados. Los participantes son socios de la asociación AIPO (Asociación Española de Interacción Persona-Ordenador) y pertenecen a distintas disciplinas (arquitectura de la información, diseño gráfico, informática educativa, ingeniería del software e interacción persona-ordenador). Se pidió a cada uno que clasificara distintos atributos incluidos en una lista simplificada del conjunto de atributos de reacciones a un producto software (The complete set of 118 product reaction cards) propuesta por Microsoft en 2002. Concretamente, el subconjunto utilizado suprimía antónimos y atributos con carácter negativo, quedando únicamente aquellos que aportaban un aspecto positivo desde el punto de vista del usuario. En la figura 5 se han recogido aquellos sobre los que ha existido mayor consenso en su clasificación.

Con esta clasificación de atributos se ha evidenciado que los profesionales consultados, conforme crece la consideración del usuario y el nivel de interacción, utilizan mayoritariamente los conceptos más recientes en el ámbito de la calidad de interacción: calidad en uso y experiencia de usuario. Así, usabilidad o user-friendliness no resultan suficientes para aglutinar muchos de esos atributos, aunque siguen siendo utilizados para hacer referencia a características más directamente relacionadas con el sistema en sí.

\section{La usabilidad mide y evalúa los objeti- vos pragmáticos, mientras que la UX se centra en la evaluación de los objetivos hedónicos}

Se ha analizado cómo la calidad de la interacción se ha ido haciendo un hueco en la literatura. En este sentido, se han identificado publicaciones científicas relacionadas, apreciando una evolución en número e índice de calidad de las mismas. Se realizó un análisis bibliométrico de los últimos veinte años en fuentes de datos como DBLP (base de datos 


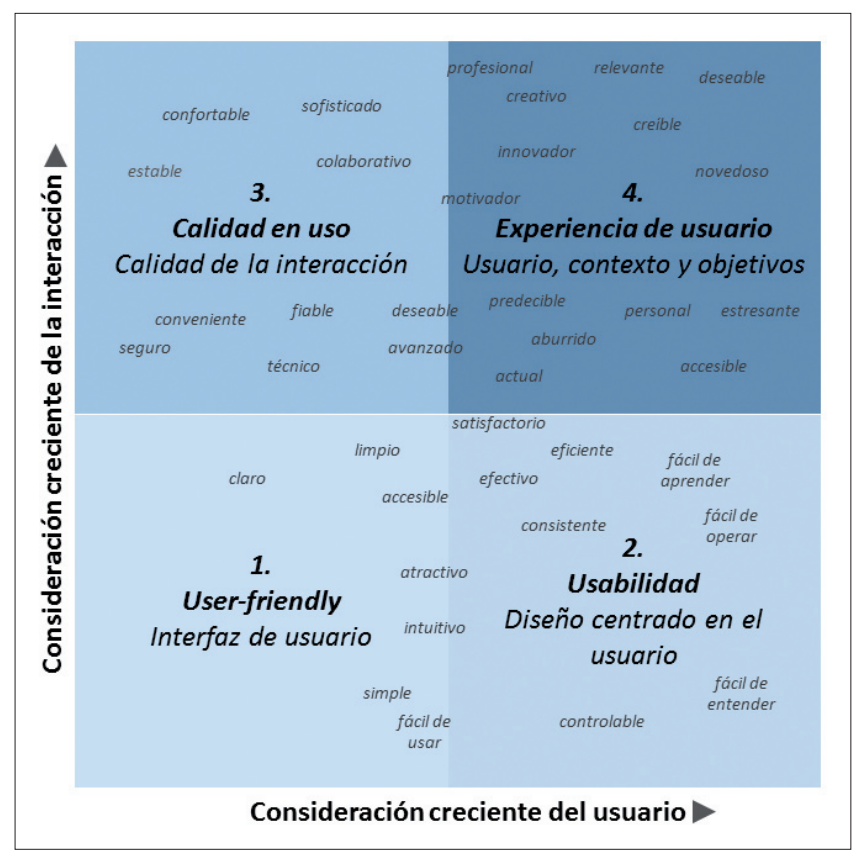

Figura 5. De la interfaz de usuario a la experiencia de usuario

de informática de la Universität Trier) e ISI Web of Science. En la figura 6 se muestra cómo la usabilidad ha sido un tema recurrente en la investigación del proceso interactivo dentro de disciplinas como la informática y la arquitectura de la información, copando el mayor número de publicaciones. En los últimos cinco años ha comenzado a descender la presencia del concepto de usabilidad en los artículos y han aumentado las publicaciones que hacen referencia a otros conceptos descritos en este artículo, como la calidad en uso o la experiencia de usuario. Estos valores son orientativos, ya que dado el carácter multifacético de la experiencia de usuario, y que la usabilidad es una parte importante de esta, es difícil separarlas. Para realizar el estudio se han buscado por título o palabras clave algunos de los identificadores de calidad presentados en este trabajo.

\section{Análisis y conclusiones}

Las posibilidades y facilidades de interacción de un sistema de información son un elemento clave a la hora de elegir, aceptar, desarrollar y formarse una opinión sobre dicho sis- tema. Sin embargo tradicionalmente esa cualidad del software no ha sido una calidad en sí sino una característica o factor de calidad. Conceptos como el de interfaz amigable (user friendly) o el de usabilidad son los que se han venido utilizando para hacer referencia a esas características del producto y de sus cualidades.

Recientemente dos estándares internacionales de la Organización Internacional de Estandarización (ISO), como la ISO/IEC 9241-210:2010 y la ISO/IEC 25010:2011, han enfatizado el aspecto interactivo de los sistemas a través de las consideraciones ergonómicas de la interacción y la identificación de la calidad en uso de los sistemas de información. En el concepto de experiencia de usuario (UX) tienen cabida otras características, tanto internas como externas, de los sistemas de información, incluyendo entre otros aspectos aquellos relacionados con su credibilidad, su valor y su utilidad. Debido al aumento de software o de sistemas de información existentes capaces de realizar la misma tarea y la multitud de opciones existentes para el usuario, el concepto de usabilidad como principal indicador de calidad queda solapado por otro aspecto mucho más amplio como es la experiencia de usuario, que intenta profundizar dentro de la satisfacción del usuario para que las sensaciones del proceso de uso sean las mejores.

\section{El objetivo no es sólo que nuestros pro- ductos sean rápidos eficaces y eficien- tes, se intenta que el usuario se sienta satisfecho usándolos}

Todos estos conceptos ligados a los sistemas de información se usan para describir las características de esos sistemas, pero deben conocerse el alcance y dimensión que cada uno aporta. En este artículo se han recogido referencias, definiciones, caracterizaciones y retos que presentan la descripción y definición de la calidad de la interacción ofrecida por un producto software.

La calidad de la interacción influye de manera decisiva en que los usuarios se decanten por un sistema u otro. Los principales desafíos que presenta la definición de la calidad
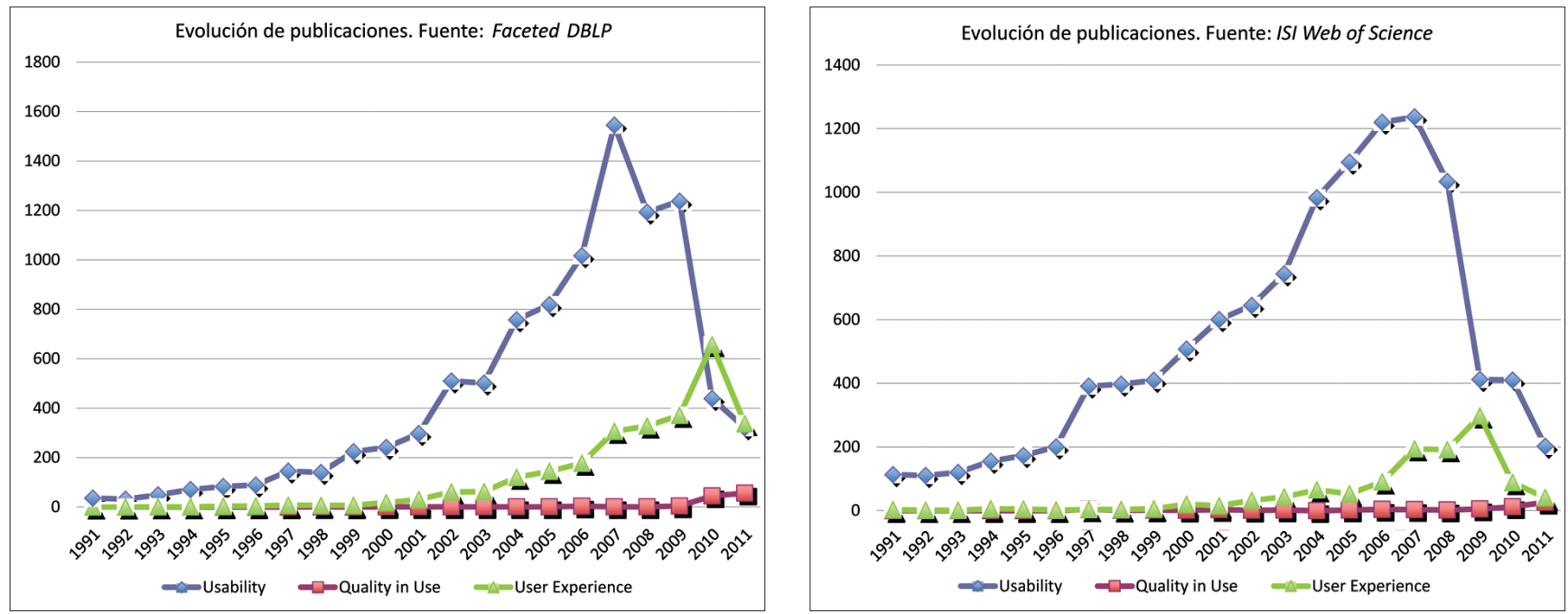

Figura 6. Número de publicaciones sobre indicadores de calidad tratados desde 1991 a 2011 
de la interacción en estos momentos están en la necesidad de considerar las propiedades hedónicas del proceso interactivo. En ese ámbito identificamos la necesidad de tener en cuenta en los conceptos y definiciones disponibles otros elementos como son las emociones, la percepción de los estímulos y el análisis de las reacciones del usuario ante dichos estímulos (reacciones viscerales, complacencia, placer, awareness -concienciación, sensibilización-, etc.).

Sin duda estamos ante un cambio "generacional" donde el objetivo no sólo está en que nuestros productos sean rápidos, eficaces y eficientes o la información esté bien diseñada y sea de fácil acceso. Se tiende a buscar ese valor añadido que es que el usuario se sienta satisfecho (placer, felicidad, seguridad, fidelidad, etc.), provocando que la calidad del proceso de uso sea la mejor posible.

\section{Bibliografía}

Baeza-Yates, Ricardo; Rivera-Loaiza, Cuauhtémoc; VelascoMartín, Javier. "Arquitectura de la información y usabilidad en la web". El profesional de la información, 2004, v. 13, n. 3, pp. 169-178.

http://www.elprofesionaldelainformacion.com/conteni dos/2004/mayo/1.pdf

http://dx.doi.org/10.1080/13866710412331291886

Basili, Victor; Heidrich, Jens; Lindvall, Mikael; Münch, Jürgen; Regardie, Myrna; Trendowicz, Adam. "GQM+ strategies. Aligning business strategies with software measurement". Proceedings of ESEM, 2007, pp. 488-90.

http://www.cs.umd.edu/ basili/publications/proceedings/ P122.pdf

Bastien, J. M. Christian; Scapin, Dominique. "Evaluating a user interface with ergonomic criteria". Intl journal of human-computer interaction, 1995, v. 7, n. 2, pp. 105-121. http://dx.doi.org/10.1080/10447319509526114

Bevan, Nigel. "Quality in use: meeting user needs for quality". Journal of system and software, 1999a, v. 49, n. 1, pp. 89-96.

http://www.nigelbevan.com/papers/qiuse.pdf http://dx.doi.org/10.1016/S0164-1212(99)00070-9

Bevan, Nigel. "Quality in use for all". In: User Interfaces for All. En: Stephanidis, Constantine (ed.). User interfaces for all. Lawrence Erlbaum, 1999b. ISBN: 9780805829679

http://www.nigelbevan.com/papers/quality_in_use_for_ all.pdf

Boehm, Barry; Brown, John R.; Kaspar, Hans; Lipow, Myron; McLeod, Gordon J.; Merritt, Michael J. Characteristics of software quality. The Netherlands: North Holland Publishing, 1978. ISBN: 0444851054

Cockton, Gilbert. "Putting value into E-valuation". En: Law, Effie; Hvannberg, Ebba; Cockton, Gilbert (eds.). Maturing usability: quality in software, interaction and value. London: Springer Verlag, 2008. ISBN: 9781846289408

Constantine, Larry L.; Lockwood, Lucy A. D. Software for use: a practical guide to the models and methods of usage-centered design. Addison-Wesley, 1999. ISBN: 978 0321773722
Dromey, R. Geoff. "A model for software product quality". IEEE transactions on software engineering, 1996, v. 21, n. 2, pp. 146-162.

http://dx.doi.org/10.1109/32.345830

Garrett, Jesse-James. The elements of user experience: usercentered design for the Web and beyond. $2^{\text {nd }}$ ed. New Riders Press, 2010. ISBN: 9780321683687

Hassenzahl, Marc. "The thing and I: understanding the relationship between user and product". En: Blythe, Mark; Monk, Andrew; Overbeeke, Kees; Wright, Peter C. Funology: from usability to enjoyment. Dordrecht: Kluwer Academic Publishers, 2003, pp. 31-42. ISBN: 9781402012525

http://www.uni-landau.de/hassenzahl/pdfs/03 Hassenzahl. $p d f$

Hofstede, Geert; Hofstede, Gert-Jan. Cultures and organizations: software of the mind. New York: McGraw-Hill, 2005. ISBN: 0071439595

ISO 9241-11:1998. Ergonomic requirements for office work with visual display terminals (VDTs). Part 11: Guidance on usability, 1998.

http://www.it.uu.se/edu/course/homepage/acsd/vt09/ ISO9241part11.pdf

ISO/IEC 9241-210:2010. Ergonomics of human-system interaction. Part 210: Human centred design for interactive systems. Clausule 2.15, 2010.

ISO/IEC 25000:2005. Software engineering. Software product quality requirements and evaluation (SQuaRE). Guide to SQUaRE, 2005.

ISO/IEC 25010:2011. Systems and software engineering. Systems and software quality requirements and evaluation (SQuaRE). System and software quality, 2011.

ISO/IEC 9126-1:2001. Software engineering. Product quality. Part 1: Quality model, 2001.

ISO/IEC 9126:1991. Software enginnering. Product quality, 1991.

ISO/IEC TR 9126-2:2003. Software engineering. Product quality. Part 2: External metrics, 2003.

ISO/IEC TR 9126-3:2003. Software engineering. Product quality. Part 3: Internal metrics, 2003.

ISO/IEC TR 9126-4:2004. Software engineering. Product quality. Part 4: Quality in use metrics, 2004.

Krug, Steve. Don't make me think: a common sense approach to web usability. $2^{\text {nd }}$ ed. New Riders Press, 2005. ISBN: 9780321344755

Law, Effie-Lai-Chong. "The measurability and predictability of user experience". En: Proceedings of EICS 2011, pp. 1-10.

Law, Effie-Lai-Chong; Roto, Virpi; Hassenzahl, Marc; Vermeeren, Arnold P.O.S.; Kort, Joke. "Understanding, scoping and defining user experience: a survey approach". Proceedings of CHII. User experience, 2009, pp. 719-28.

http://www.itu.dk/ jeppeh/specialeartikler/Understand ing, Scoping and Defining User eXperience.pdf 
Law, Effie-Lai-Chong; Roto, Virpi; Vermeeren, Arnold P.O.S.; Kort, Joke; Hassenzahl, Marc. "Towards a shared definition of user experience". En: Proceedings of CHI'08, Extended abstracts on human factors in computing systems, 2008, pp. 2395-2398. ISBN: 9781695580128

http://dx.doi.org/10.1145/1358628.1358693

Law, Effie-Lai-Chong; Van-Schaik, Paul. Modelling user experience. An agenda for research and practice. Interacting with computers, 2010 , v. 22, n. 5, pp. 313-22.

http://dx.doi.org/10.1016/j.intcom.2010.04.006

Marcos, Mari-Carmen. "Percibir, procesar y memorizar". El profesional de la información, 2004, v. 13, n. 3, pp. 197-202. http://www.elprofesionaldelainformacion.com/conteni dos/2004/mayo/4.pdf

http://dx.doi.org/10.1080/13866710412331291916

Mayhew, Deborah. The usability engineering lifecycle: $a$ practitioner's handbook for user interface design (interactive technologies). Morgan Kaufmann, 1999. ISBN: 978 1558605619

McCall, Jim A.; Richards, Paul K.; Walters, Gene F. Factors in software quality. v. I, II y III. US Rome Air development center reports, 1977.
Nielsen, Jacob. Usability engineering. $1^{\text {st }}$ ed. Morgan Kaufmann, 1993. ISBN: 9780125184069

Norman, Donald. The design of everyday things. Basic Books, 2002. ISBN: 9780385267748

Norman, Donald. The design of future things. Basic Books, 2009. ISBN: 9780465002276

Pressman, Roger S. "Development strategies and project management". The computer science and engineering handbook, 1997, pp. 2399-2418.

Ribera, Mireia; Térmens, Miquel; García-Martín, Maika. "Cómo realizar tests de usabilidad con personas ciegas". El profesional de la información, 2008, v. 17, n. 1, pp. 99-105. http://www.elprofesionaldelainformacion.com/contenidos/2008/enero/17.pdf

http://dx.doi.org/10.3145/epi.2008.ene.12

Vermeeren, Arnold P.O.S.; Law, Effie-Lai-Chong; Obrist, Marianna; Hoonhout, Jettie; Väänänen-Vainio-Mattila, Kaisa. "User experience evaluation methods: current state and development needs". Proceedings of NordiCHI'2011, pp. 521-30.

http://dx.doi.org/10.1145/1868914.1868973
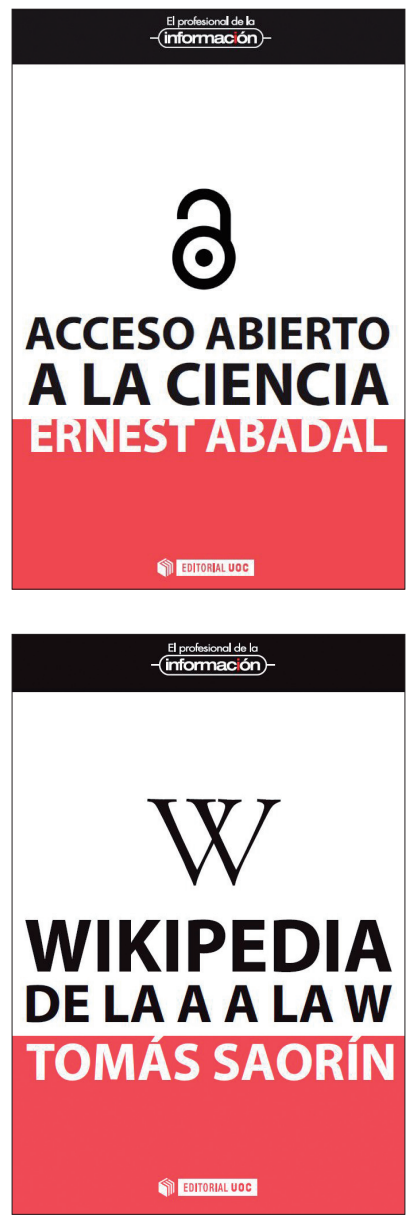
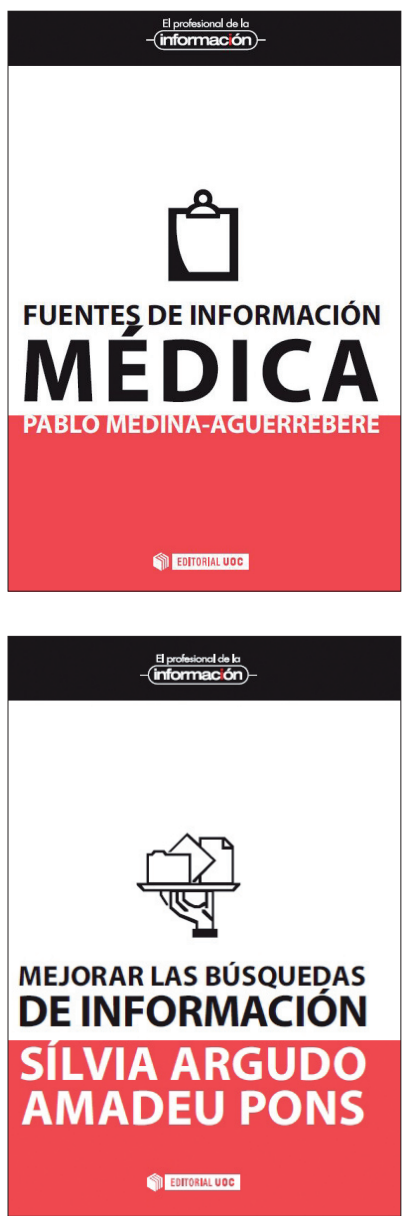
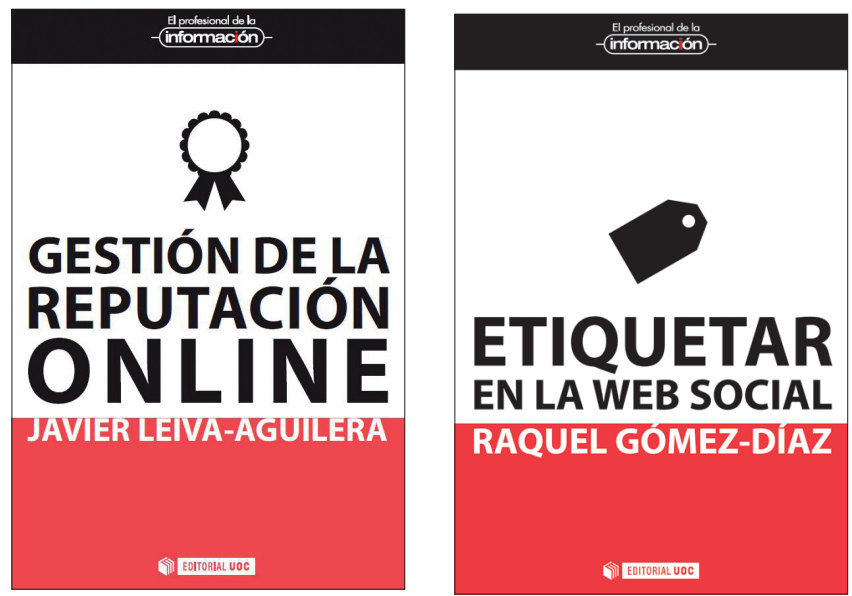

\section{Colección de libros} de bolsillo
EPI-UOC

Más información:

http://www.elprofesionaldelainformacion.com/libros.html 